\title{
Haemostatic Nanoparticles-Derived Bioactivity of from Selaginella tamariscina Carbonisata
}

\author{
Yusheng Zhao ${ }^{1}$, Yue Zhang ${ }^{2}$, Hui Kong ${ }^{3}$, Meiling Zhang ${ }^{3}$, Jinjun Cheng ${ }^{3}$, Juan Luo ${ }^{3}$, \\ Yan $\mathrm{Zhao}^{3}$ and Huihua $\mathrm{Qu}{ }^{4, *}$ \\ 1 School of Chinese Materia Medica, Beijing University of Chinese Medicine, Beijing 100029, China; \\ 16688091339@163.com \\ 2 School of Life Sciences, Beijing University of Chinese Medicine, Beijing 100029, China; \\ 201801024@bucm.edu.cn \\ 3 School of Traditional Chinese Medicine, Beijing University of Chinese Medicine, Beijing 100029, China; \\ doris7629@126.com (H.K.); 18811790361@163.com (M.Z.); carlosjjcheng@163.com (J.C.); \\ luojuan1010@163.com (J.L.); zhaoyandr@163.com (Y.Z.) \\ 4 Centre of Scientific Experiment, Beijing University of Chinese Medicine, Beijing 100029, China \\ * Correspondence: quhuihuadr@163.com; Tel.: +86-010-6428-6705; Fax: +86-010-6428-6821
}

Received: 29 November 2019; Accepted: 14 January 2020; Published: 21 January 2020

\begin{abstract}
High-temperature carbonisation is used to prepare many traditional Chinese medicine charcoal drugs, but the bioactive haemostatic substances of these medicines and their mechanisms are still unknown. This study developed and evaluated nanoparticles (NPs) derived from Selaginella tamariscina Carbonisata (STC) for the first time. The haemostatic effect of STC-NPs prepared at 300, 350, and $400{ }^{\circ} \mathrm{C}$ were investigated in mouse tail amputation and liver scratch experiments. STC-NPs obtained at $400^{\circ} \mathrm{C}$ had the strongest haemostatic effect, and were accordingly characterised by ultraviolet-visible spectroscopy, fluorescence spectroscopy, Fourier transform infrared spectroscopy, transmission electron microscopy, high resolution transmission electron microscopy, X-ray diffractometry, and X-ray photoelectron spectroscopy. STC-NPs averaged $1.4-2.8 \mathrm{~nm}$ and exhibited a quantum yield of $6.06 \%$ at a maximum excitation wavelength of $332 \mathrm{~nm}$ and emission at $432 \mathrm{~nm}$. STC-NPs displayed low toxicity against mouse monocyte macrophage RAW 264.7 cells by CCK-8 assay, and STC-NP treatment significantly shortened bleeding time in rat and mouse models. Coagulation assays showed that the haemostatic effects of STC-NPs were related to improving the fibrinogen and platelet contents, as well as decreasing the prothrombin time that resulted from stimulating extrinsic blood coagulation and activating the fibrinogen system. The STC-NPs had remarkable haemostatic effects in the tail amputation and liver scratch models; these effects may be associated with the exogenous coagulation pathway and activation of the brinogen system, according to the evaluation of the mouse coagulation parameters. This novel evaluation supports the material basis of STC use in traditional Chinese medicine, and this article is worthy of study by authors of clinical pharmacy.
\end{abstract}

Keywords: Selaginella tamariscina carbonisata; nanoparticles; haemostasis

\section{Introduction}

Charcoal treatment is a major category of Chinese medicine, with 2000 years of historical application, especially in the treatment of haemorrhagic diseases that show a convergent haemostatic effect [1-3]. The haemostatic effect of charcoal has been included in the Chinese Pharmacopoeia [4]. Modern pharmacological studies [5] have proven that Schizonepetae herba carbonisata can increase the FIB and platelet content in rats to achieve haemostatic purposes. High-temperature carbonisation is a critical process in the preparation of traditional Chinese medicine (TCM) charcoal formulations. 
However, the bioactive haemostatic substances of charcoal medicine and their mechanisms have not been clearly reported. One study [6] showed that tannins play a major role in the haemostatic effect of carbon drugs. Another study reported that it was a change in flavonoids that was closely related to haemostatic effect enhancement [7]. These inconsistent results cannot reasonably explain the similar haemostatic effects of many different charcoal medicines. We tried to clarify which small molecular compounds were responsible for the effects of Selaginella tamariscina Carbonisata (STC), but the results have been uncertain. STC was not detected in the small molecules after carbonization and dialysis. Therefore, this study explains the haemostatic effect of carbonized medicinal materials from the perspective of nanomaterials.

Selaginella tamariscina (ST), locally called "Juan Bai", is widely distributed in China. The Pharmacopoeia of the People's Republic stipulates that ST is the all dry grass of Selaginella tamariscina (Beauv.) Spring or Selaginella pulvinata (Hook. Et Grev.) Maxim. ST has been widely used in China as a medicine to improve blood circulation. ST was first recorded as a medicinal plant in ShengNong's Herbal Classic, written more than 2000 years ago. However, its processed product, Selaginella tamariscina Carbonisata (STC), possesses opposing haemostatic activity and is clinically used as a TCM haemostatic to treat haematemesis, disintegration, haematochezia, and other bleeding symptoms. Modern medical research [8] has also confirmed that STC is an effective haemostatic after carbonisation. Carbon dots are nanoparticles, usually 1-10 $\mathrm{nm}$ in diameter, with proven theranostic activity [9]. In recent years, under the existing concepts of the material basis for traditional Chinese medicine, many scholars $[6,10]$ have attempted to gain an understanding of the haemostatic material foundation of small molecules or metal ions from the perspective of Chinese medicine, but no obvious research progress has been made. Therefore, the haemostatic components of charcoal drugs must be determined. Nanoparticles have been reported [11] to have haemostatic biological activity. The nanotechnology used in this research shows great clinical medicinal value, which provides evidence to support the further investigation of the considerable potential haemostatic drug and effective material basis of TCM.

STC is a type of calcined herb drug that has been used as a haemostatic medicine to promote haemostasis for thousands of years. This study aimed to explore the haemostatic effect of STC and used STC as an example by which to clarify the novel substances generated after the charcoal processing of Selaginella tamariscina. By evaluating the physicochemical properties, such as morphological size, elemental components, and functional groups, using a variety of analytical techniques, this study elucidates the differences between ST and STC, in particular, the STC nanoparticles (STC-NPs) created and evaluated herein. The effect of STC-NPs on key parameters involved in haemorheological and coagulation systems were investigated using rat and mouse models.

\section{Results and Discussion}

\subsection{Preparation Temperature Optimisation}

The preparation temperature conditions of STC-NPs were optimised using three different temperatures (i.e., $300^{\circ} \mathrm{C}, 350^{\circ} \mathrm{C}$, and $400^{\circ} \mathrm{C}$ ). As shown in Figure $1 \mathrm{a}$, compared to the NS group, the positive group and the STC-NPs groups prepared at different temperatures had haemostatic effects in the tail amputation model. Furthermore, in the liver scratch model, the positive group and the STC-NP groups significantly decreased the liver bleeding time (Figure 1b). The bleeding time of mice intraperitoneally injected with STC-NPs prepared at different temperatures ranged from short to long $\left(300^{\circ} \mathrm{C}<350{ }^{\circ} \mathrm{C}<400{ }^{\circ} \mathrm{C}\right)$. STC-NPs prepared at $400{ }^{\circ} \mathrm{C}$ had the strongest haemostatic effect (significantly shortened bleeding time) and were selected for use in the following experiments. 

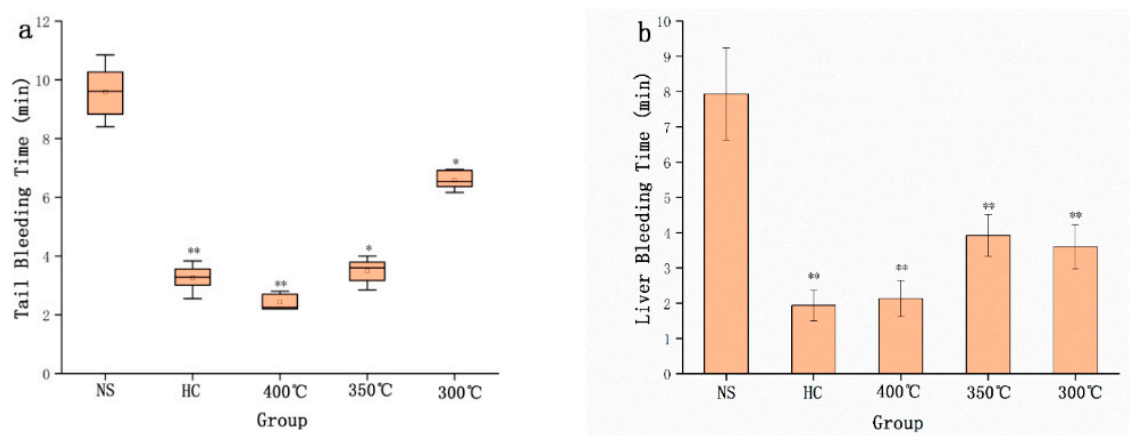

Figure 1. Haemostatic time in mouse tail amputation and liver scratch models: (a) Tail amputation $(n=8)$ and $(\mathbf{b})$ liver scratch $(n=8)$ models treated with normal saline (NS), haemocoagulase (HC), and STC-NPs produced at $400{ }^{\circ} \mathrm{C}, 350{ }^{\circ} \mathrm{C}$, and $300{ }^{\circ} \mathrm{C} .{ }^{* *} p<0.01$ and ${ }^{*} p<0.05$ compared to the control group $(n=8)$.

\subsection{High-Performance Liquid Chromatography Data Analysis}

The chromatogram obtained from the methanol extract of ST contained several compounds, such as amentoflavone (see Figure 2a). However, no significant peaks were observed in the aqueous STC solution (see Figure $2 \mathrm{~b}$ ), indicating that no active small molecule compounds of the ST were detected in the obtained STC-NPs solution [12]. The results of this experiment show that the samples were highly purified by charcoal processing and dialysis.

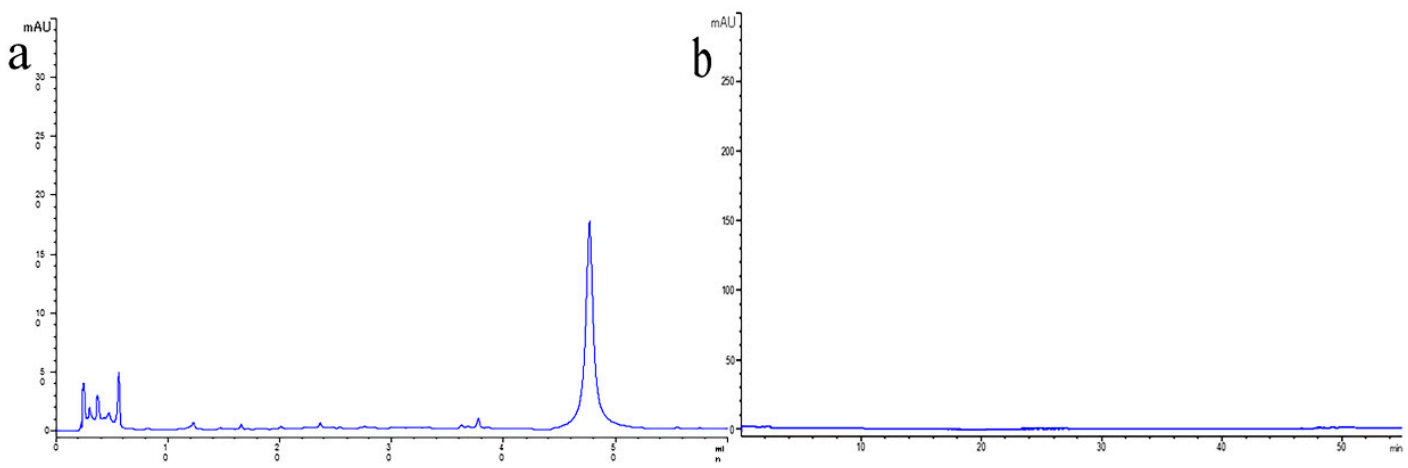

Figure 2. High-performance liquid chromatography (HPLC) fingerprint of (a) ST (the all dry grass of Selaginella tamariscina (Beauv.) Spring) and (b) Selaginella tamariscina Carbonisata.

\subsection{Characterisation of STC-NPS}

As shown in Figure 3a, the TEM microscope image of STC-NPs indicated a uniformly distributed particle size from $1.4 \mathrm{~nm}$ to $2.8 \mathrm{~nm}$ that was mainly concentrated at approximately $2.0-2.2 \mathrm{~nm}$; the distribution conformed to normal distribution characteristics. The HRTEM results showed that the STC-NP particle was a spherical structure, with well-resolved lattice fringes and a lattice spacing of $0.381 \mathrm{~nm}$ (see Figure $3 \mathrm{~b}$ ). This spacing corresponds to that in previously published literature $(0.390 \mathrm{~nm})$ [13]. Figure 3c shows that the XRD spectrum of STC-NPs had distinct diffraction peaks $\left(2 \theta=22.765^{\circ}\right)$, indicating that STC-NPs were composed of highly amorphous carbon structures [14].

The NP optical properties were characterised using UV-vis and fluorescence spectroscopy. As is shown in Figure 3d, the NPs had a weak absorption peak at approximately $260 \mathrm{~nm}$, corresponding to the $\pi-\pi^{*}$ transition of the conjugated $C=C$ bonds and aromatic sp2 domains $[15,16]$. The fluorescence spectrum of STC-NPs showed a maximum emission wavelength at approximately $432 \mathrm{~nm}$ and excitation wavelength at $332 \mathrm{~nm}$ (Figure 3e). The QY of STC-NPs was calculated to be $6.06 \%$ using quinine sulphate as a reference. The FT-IR spectrum was used to determine NP surface functional groups (Figure 3f). NPs exhibited a broad characteristic peak at $3441 \mathrm{~cm}^{-1}$ corresponding to the -O-H stretching vibration. The characteristic absorption at $\left(\mathrm{cm}^{-1}\right)$ 2921, 2860, and 1400 indicated $-\mathrm{CH}_{3}$ (stretching), 
$-\mathrm{CH}_{2}$ (stretching), and the stretching vibration peak of $\mathrm{C}-\mathrm{N}$, respectively. In addition, NPs displayed the characteristic bands for $\mathrm{C}=\mathrm{O}$ and $\mathrm{C}-\mathrm{O}-\mathrm{C}$ vibrations at $1633 \mathrm{~cm}^{-1}$ and $1048 \mathrm{~cm}^{-1}$, respectively, thereby indicating the presence of $\mathrm{sp}^{2}[17,18]$. It was concluded that the STC-NP surface contained carbonyl, hydroxyl, and amidogen functional groups.
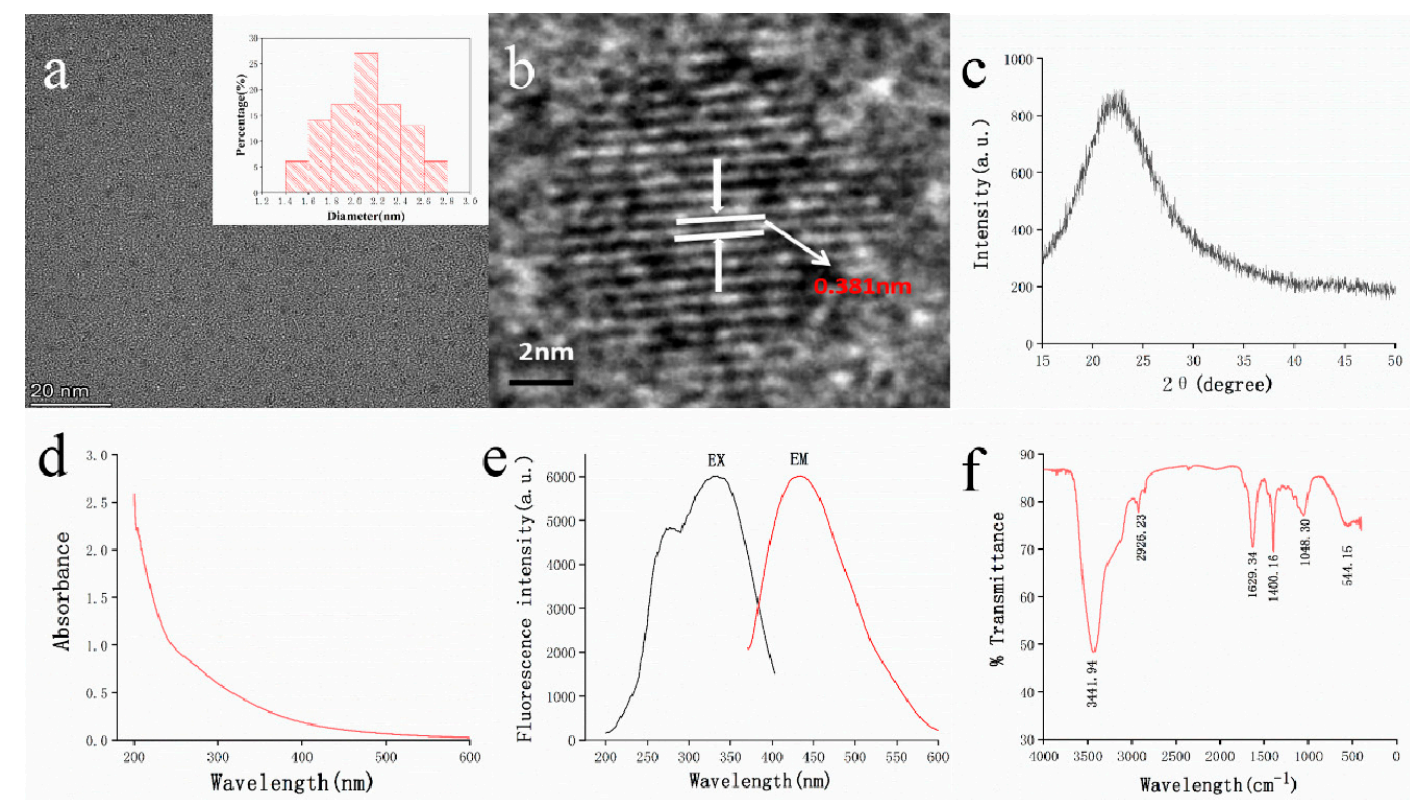

Figure 3. Characterisation of STC-NPs: (a) Transmission electron microscopy (TEM) images of STC-NPs displaying ultra-small particles and TEM size distribution of STC-NPs (Upper right corner). (b) High-resolution TEM image of STC-NPs. (c) X-ray diffraction pattern. (d) Ultraviolet-visible spectrum. (e) Fluorescence spectrum. (f) Fourier transform infra-red spectrum.

The surface and elemental composition of STC-NPs were further studied by X-ray photoelectron spectroscopy (XPS). As shown in Figure 4a, peaks were evident at 400.41 and $532.95 \mathrm{eV}$, indicating that the quantum point was composed mainly of O $(87.21 \%), \mathrm{C}(11.25 \%)$, and a small amount of $\mathrm{N}(1.54 \%$ ). The XPS spectrum of O1s (Figure $4 \mathrm{~b}$ ) was fitted with two peaks at 532.6 and $533.4 \mathrm{eV}$, which were attributed to $\mathrm{C}-\mathrm{OH}$ and $\mathrm{O}-\mathrm{C}=\mathrm{O}$, respectively. The $\mathrm{C} 1 \mathrm{~s}$ spectrum (Figure $4 \mathrm{c}$ ) was divided into three peaks at $284.8,286.1$, and $288.2 \mathrm{eV}$, which were assigned to $\mathrm{C}-\mathrm{OH}, \mathrm{C}=\mathrm{O}$, and $\mathrm{C}=\mathrm{N}$ bonds, respectively. The N1s spectrum (Figure 4d) was resolved into two peaks at 399.8 and $401.2 \mathrm{eV}$. These peaks corresponded to $\mathrm{C}-\mathrm{N}-\mathrm{C}$ and $(\mathrm{C}) 3-\mathrm{N}$ bonds, respectively $[19,20]$. The experimental results were consistent with the FT-IR characterisation [21,22]. Our FT-IR and XPS analysis showed that STC-NPs contain hydroxyl groups, and that the presence of carbonyl groups can be attributed to the multiphoton activity process from various oxygen-containing functional groups [23].

\subsection{Cytotoxicity Detection}

NP toxicity has always been an essential problem in biological application. The safety of STC-NPs was evaluated by detecting the cell viability of RAW 264.7 cells in a CCK-8 assay. As shown in Figure 5, the STC-NPs were not toxic to cells at concentrations up to $1.25 \mathrm{mg} / \mathrm{mL}$. A concentration of $10 \mathrm{mg} / \mathrm{mL}$ decreased RAW 264.7 cell viability. The experimental results showed that the STC-NPs were safe and biocompatible at concentrations up to $1.25 \mathrm{mg} / \mathrm{mL}$. 

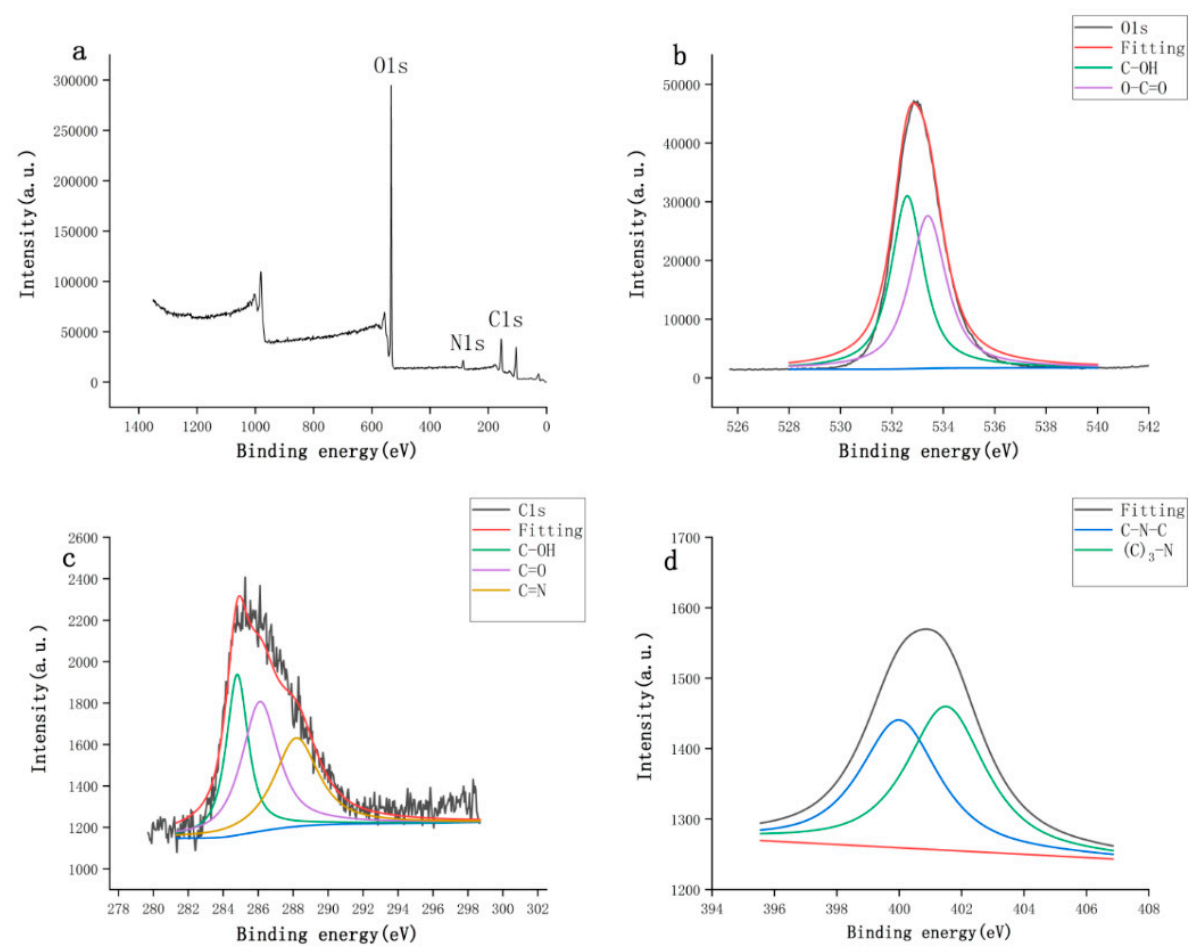

Figure 4. The surface composition and elemental analysis of the prepared STC-NPs by XPS. (a) X-ray photoelectron spectroscopy survey of STC-NPs. (b) C1s. (c) O1s and (d) N1s.

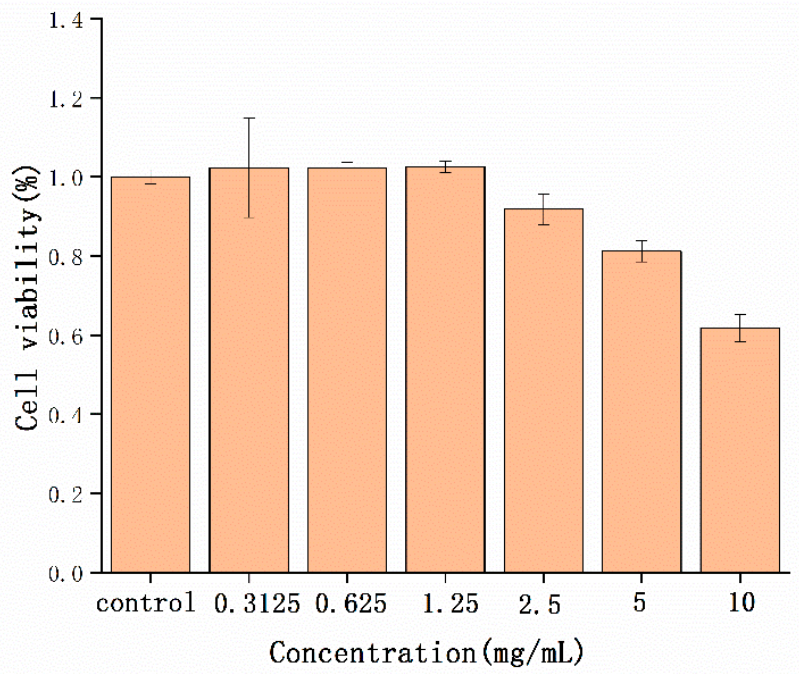

Figure 5. Effect of different concentrations of STC-NPs on the viability of RAW 264.7 cells.

\subsection{Haemostatic Effect of STC-NPS}

Compared to the NS group $(9.07 \pm 0.67 \mathrm{~min})$, the HC group $(2.33 \pm 0.31 \mathrm{~min})$ and the high-, medium- and low-dose STC-NPs groups $(2.75 \pm 0.62,1.82 \pm 0.26,3.59 \pm 0.41 \mathrm{~min}$, respectively) significantly $(p<0.01)$ reduced tail bleeding time (Figure 6a). Liver bleeding time was the longest in the NS group $(7.93 \pm 0.55 \mathrm{~min})$. Figure $6 \mathrm{~b}$ shows that both the positive group $(2.68 \pm 0.39 \mathrm{~min})$ and the STC-NP groups (H $2.46 \pm 0.29, \mathrm{M} 2.08 \pm 0.27, \mathrm{~L} 3.61 \pm 0.33 \mathrm{~min})$ significantly $(p<0.01)$ reduced liver bleeding time compared to the normal saline group. These results indicated that the STC-NPs had a significant haemostatic effect. 

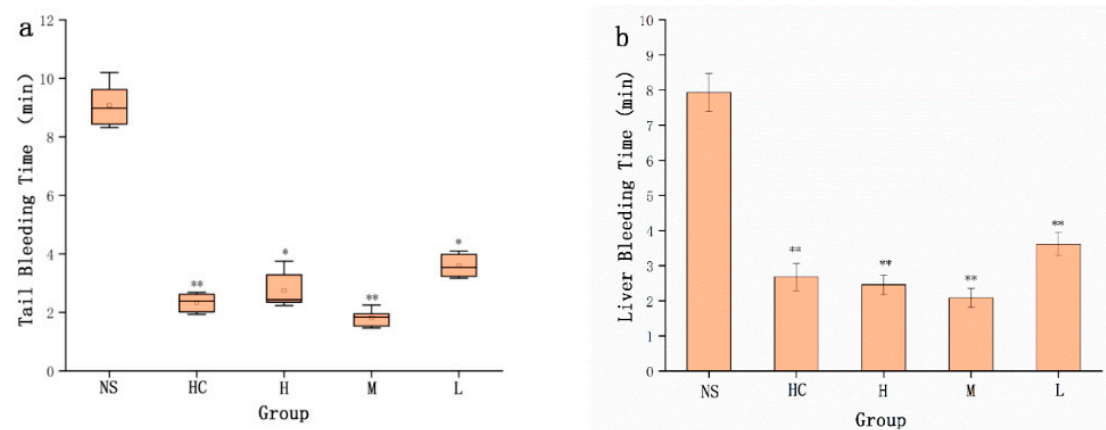

Figure 6. Graphs of haemostatic time in mouse tail amputation and liver scratch models. (a) Tail amputation $(n=8)$ and $(\mathbf{b})$ liver scratch $(n=8)$ models treated with normal saline (NS), haemocoagulase $(\mathrm{HC})$, and high $(\mathrm{H})$, medium $(\mathrm{M})$, and low $(\mathrm{L})$ doses of STC-NPs $(16.67,8.33$, and $4.17 \mathrm{mg} / \mathrm{kg}$, respectively). ${ }^{* *} p<0.01$ and ${ }^{*} p<0.05$ compared to the control group $(n=8)$.

\subsection{Effect of STC-NPs on the Coagulation System}

The haemostatic mechanism of STC-NP activity evaluated by measuring four coagulation parameters (i.e., activated partial thromboplastin time (APTT), thrombin time (TT), prothrombin time (PT), and fibrinogen content (FIB), and platelet counts (PLT)) in rats showed that APTT and TT values were not significantly different among the four treatment groups (Figure 7a,b). As shown in Figure 7c, compared to the NS group, PT values in HC and the STC-NP groups were significantly decreased $(\mathrm{p}<0.05)$. Fibrinogen (FIB) is an important index by which to study the coagulation process. Figure $7 \mathrm{~d}$ shows that the high-, medium-, and low-dose STC-NPs groups and HC groups showed significantly $(p<0.01)$ increased FIB values $(2.29 \mathrm{~g} / \mathrm{L}, 2.35 \mathrm{~g} / \mathrm{L}, 2.28 \mathrm{~g} / \mathrm{L}$, and $2.30 \mathrm{~g} / \mathrm{L}$, respectively) compared to that of the NS group $(2.04 \mathrm{~g} / \mathrm{L})$. The number of platelets (PLT) closely reflected the coagulation situation, and STC-NPs had a significant effect on PLT in rats. Compared to the NS group $\left(1053 \times 10^{9} / \mathrm{L}\right)$, the high-, medium-, and low-dose STC-NPs groups and the HC group $\left(1151 \times 10^{9} / \mathrm{L}, 1142 \times 10^{9} / \mathrm{L}, 1121 \times 10^{9} / \mathrm{L}\right.$, and $1136 \times 10^{9} / \mathrm{L}$, respectively) significantly $(p<0.05)$ increased PLT in rats (Figure $\left.7 \mathrm{e}\right)$.
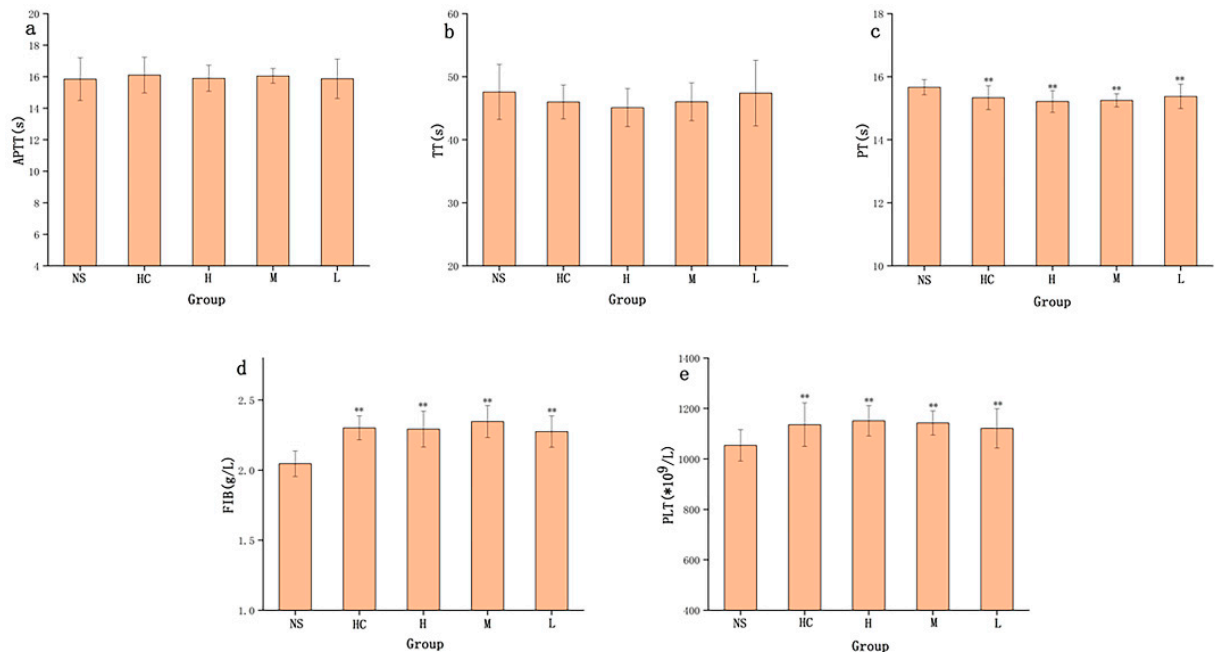

Figure 7. Effects on coagulation parameters. (a) Activated partial thromboplastin time (APTT), (b) thrombin time (TT), (c) prothrombin time (PT), (d) fibrinogen (FIB), and (e) platelet count (PLT). Analysis of rats treated with normal saline (NS), haemocoagulase $(\mathrm{HC})$, or high $(\mathrm{H})$, medium $(\mathrm{M})$, or low (L) doses of STC-NPs $\left(16.67,8.33\right.$, and $4.17 \mathrm{mg} / \mathrm{kg}$, respectively) ${ }^{* *} p<0.01$ and ${ }^{*} p<0.05$ compared to control group $(n=10)$.

Nanoparticles have many unique properties such as high aqueous solubility, photoluminescence, straightforward functionalisation, and biocompatibility [24,25]. A range of applications has been 
developed for emerging nanomaterials including drug delivery [26], biological imaging [27], and optical catalysts [28]. NPs have been reported in other TCMs carbonised at high temperature including Pollen Typhae Carbonisata, Cirsium Setosum Carbonisata, Schizonepetae Herba Carbonisata, and Junci Medulla Carbonisata [5,29-31]. The STC-NPs possessed components similar to those of other widely-studied nanoparticles [32,33]. In the TEM images, the particle size and morphology of STC-NPs are similar to those previously reported of carbon dots [34]. It can be clearly seen that the atomic lattice fringes of the STC-NPs are consistent with that of graphene quantum dots [35]. According to the UV-vis data, the long absorption tail, i.e., up to $600 \mathrm{~nm}$, is due to the low energy transitions within the surface states, created by the surface functional groups of the STC-NPs [36]. This may be the key to affecting their chemical properties. In addition, this study used HPLC to compare the different chemical compositions of ST and STC-NPs to identify component variation between the two. The main active components of ST contain flavonoids and other small molecular substances [37]. In sharp contrast, STC-NPs did not contain small molecule compounds, which, to some extent, excludes the interference of small molecule compounds on haemostatic activity.

The normal physiological haemostasis and anticoagulation mechanism is a complex physiological and biochemical process mainly composed of three interrelated parts: vasoconstriction and platelet response, anticoagulation systems, and fibrinolysis systems [38]. The coagulation process can be roughly divided into three stages: prothrombin activator formation, thrombin formation, and fibrin formation. In the current study, STC-NPs had no effect on TT and APTT values, and therefore, did not affect the endogenous coagulation pathway in rats. However, the increased PT value indicated that STC-NPs might affect the exogenous coagulation pathway. FIB is an important indicator by which to study the coagulation process and the influence of drugs on coagulation, anticoagulation, and fibrinolytic system function [39]. We speculated that the coagulation-promoting activity of STC-NPs might play a role in haemostasis and coagulation by increasing FIB content in the blood, or by inhibiting the fibrinolytic system. STC-NPs significantly increased PLT in rats, and thereby enhanced the haemostatic effect. The haemostatic effect of STC-NPs and their related action mechanisms provide a novel idea for studying the biological activity of novel nanoparticles. The pharmacological experiment data were the most intuitive parameter to reveal the effectiveness of STC-NPs in stopping bleeding. This experiment, for the first time, proved that STC-NPs had a remarkable haemostatic effect, and it provides a preliminary experimental basis for STC-NPs to be used as a new nanoparticle-based haemostatic drug. Further investigations are needed to elucidate the underlying mechanisms of these effects. The discovery of STC-NPs and the demonstration of their haemostatic effect in this paper provide a new rationale for the material research of charcoal drugs. As an emerging nanostructure material in modern science, its pharmacological activity has been preliminary studied in this experiment. STC-NPs have the potential to become a safe and therapeutic drug for haemorrhagic diseases. This article is worthy of study by authors of clinical pharmacy.

\section{Materials and Methods}

\subsection{Materials}

ST was purchased from Beijing Qiancao Herbal Pieces Co., Ltd. (Beijing, China), and STC was prepared in our laboratory. Haemocoagulase (HC) for injection was purchased from Jinzhou Ahon Pharmaceutical Co., Ltd. (Liaoning, China). Dialysis membranes with a molecular weight of 1000 Da were purchased from Beijing Ruida Henghui Technology Development Co., Ltd. (Beijing, China). The cell counting kit (CCK)-8 was purchased from Dojindo Molecular Technologies, Inc. (Kumamoto, Japan). Mouse monocyte macrophage RAW 264.7 cells were purchased with Peking Union Cell Bank (Beijing, China). Pentobarbital sodium and other analytical grade chemical reagents were obtained from Sinopharm Chemical Reagents Beijing (Beijing, China). All experiments were performed using deionised water (DW). Male Kunming mice $(32.0 \pm 1.0 \mathrm{~g})$ and Sprague Dawley rats $(200.0 \pm 10.0 \mathrm{~g})$ were purchased from Beijing Jinmuyang Liability Co., Ltd. (Beijing, China) and kept in 
a well-ventilated room at $24.0 \pm 1.0{ }^{\circ} \mathrm{C}$ with $55-65 \%$ relative humidity and a $12 \mathrm{~h}$ light: dark cycle. The animals were given water and food ad libitum. The animal experimental design and protocols used in this study were approved by the Ethics Review Committee for Animal Experimentation at the Beijing University of Chinese Medicine. All the experimental procedures were performed in accordance with the Regulations for the Administration of Affairs Concerning Experimental Animals approved by the State Council of People's Republic of China.

\subsection{Preparation of STC-NPS}

First, $50 \mathrm{~g}$ ST was placed in a crucible and covered with aluminium foil before replacing the lid to form a seal, then calcined using a muffle furnace (TL0612, Beijing Zhong KeAobo Technology Co., Ltd., China) at 300, 350 and $400{ }^{\circ} \mathrm{C}$, respectively, for $1 \mathrm{~h}$. The obtained STC was crushed in a grinder after cooling to room temperature. Finally, the STC was dissolved in DW and boiled twice $(1 \mathrm{~h}$ each time), then filtered with filter paper to remove the residue. The solution was purified by dialysis for 7 days (molecular weight cut-off of $1.0 \mathrm{kDa}$ ), and the obtained STC-NP solution was stored at $4{ }^{\circ} \mathrm{C}$ until use. Figure 8 shows the STC-NP preparation process [5].

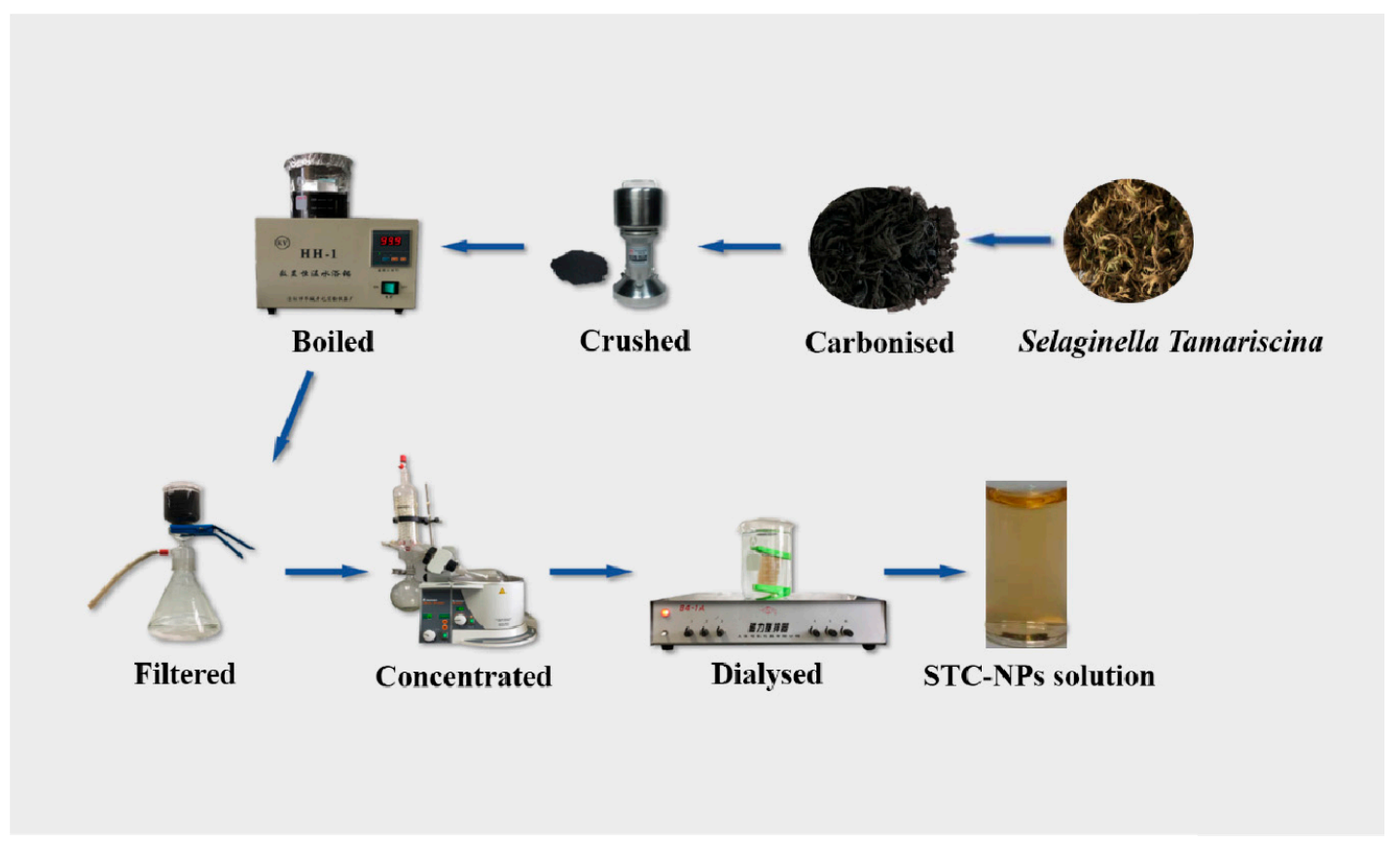

Figure 8. Flow diagram of the STC-NPs preparation process.

\subsection{Haemostatic Experiment}

The haemostatic effect of STC-NPs was evaluated using mouse tail amputation and liver scratch models. The preparation temperature conditions of STC-NPs were optimised by testing three different temperatures $\left(300^{\circ} \mathrm{C}, 350{ }^{\circ} \mathrm{C}\right.$ and $400{ }^{\circ} \mathrm{C}$ ) and the optimal preparation temperature among them was selected to determine the dose-effect relationship of the drug. In brief, mice were divided into the negative control group (normal saline (NS) intraperitoneal injection), the positive group $(0.67 \mathrm{KU} / \mathrm{kg}$ (HC) subcutaneous injection) and the high-, medium- and low-dose STC-NPs groups $(16.67,8.33$ and $4.17 \mathrm{mg} / \mathrm{kg}$, respectively, by intraperitoneal injection). The mice were anesthetised by an intraperitoneal injection of $4 \%$ pentobarbital sodium $(50 \mathrm{mg} / \mathrm{kg}) 1 \mathrm{~h}$ after treatment. In the mouse tail amputation model, clean surgical scissors were used to cut the tail $1 \mathrm{~cm}$ from the tail tip. The time from when the initial cut was made to when bleeding completely halted was recorded. In the liver scratch model, the mice were cut open along the abdominal midline with surgical scissors to expose the liver, which was then propped up with gauze soaked in saline. The left lateral lobe was punctured to a depth of $2 \mathrm{~mm}$ with a $2 \mathrm{~mL}$ syringe needle, and the wound was then overlaid with filter paper. The time 
between the onset of bleeding in the punctured liver and the cessation of bleeding was measured. The bleeding in both models was monitored at $30 \mathrm{~s}$ intervals until haemostasis was achieved.

\subsection{HPLC Analysis}

The components of the aqueous STC solution obtained at $400{ }^{\circ} \mathrm{C}$ and the methanol ST extract were measured using an Agilent 1260 series high-performance liquid chromatography (HPLC) instrument (Agilent, Waldbronn, Germany). A $\mathrm{C}_{18}$ column $(250 \mathrm{~mm} \times 4.6 \mathrm{~mm}$, Orochem, IL, USA) packed with $5 \mathrm{~mm}$ octadecyl-bonded silica $\left(\mathrm{C}_{18}\right)$ was used for STC-NP separation. All samples were filtered with a $0.22 \mu \mathrm{m}$ cellulose membrane. The mobile phases A and B were $0.1 \%$ formic acid solution and methanol, respectively. As previously reported [40], a modified gradient elution program was performed at a flow rate of $1 \mathrm{~mL} / \mathrm{min}$ as follows: $0-10 \mathrm{~min}, 10 \%-15 \%$ methanol; $10-20 \mathrm{~min}, 15-60 \%$ methanol; 20-50 $\mathrm{min}, 60-80 \%$; 50-60 $\mathrm{min}, 80-85 \%$. The column temperature was $25^{\circ} \mathrm{C}$ and the injected sample quantity was maintained at $10 \mu \mathrm{L}$. The detection wavelength was set at $330 \mathrm{~nm}$.

\subsection{Characterisation of STC-NPs}

The morphology, particle size distribution and microstructure of STC-NPs obtained at $400{ }^{\circ} \mathrm{C}$ were examined by TEM (Tecnai G220; FEI Company, USA) at $200 \mathrm{kV}$, and the lattice spacing and other internal structures were observed by HRTEM (JEN-1230; Japan Electron Optics Laboratory; Japan) and X-ray diffractometer (XRD). The optical information of STC-NPs was analysed using ultraviolet spectrophotometer (CECIL, Cambridge, UK) and fluorescence spectrophotometer (F-4500, Tokyo, Japan). An infrared spectrophotometer (Thermo, California, USA) was used to analyse the distribution of functional groups on the surface of STC-NPs from $4000-400 \mathrm{~cm}^{-1}$. The elemental and surface compositions of NPs were observed by X-ray photoelectron spectroscopy (ESCALAB 250Xi, Thermo Fisher Scientific, USA).

\subsection{Fluorescence Quantum Yield}

The fluorescence Quantum yield (QY) of the STC-NPs was measured using quinine sulphate. \% QY was 54 in $0.1 \mathrm{M}$ sulfuric acid $\left(\mathrm{H}_{2} \mathrm{SO}_{4}\right)$ solution as a standard sample. The slit widths of EX and EM were $10 \mathrm{~nm}$ and $10 \mathrm{~nm}$, respectively. In order to minimise the reabsorption effect, $A_{c}$ and $A_{q}$ remained below below 0.05 [41]. NP QY was calculated according to the following formula:

$$
\mathrm{Q}_{\mathrm{c}}=\mathrm{Q}_{\mathrm{q}}\left(\mathrm{A}_{\mathrm{c}} / \mathrm{A}_{\mathrm{q}}\right)\left(\mathrm{I}_{\mathrm{q}} / \mathrm{I}_{\mathrm{c}}\right)\left(\eta_{\mathrm{c}}{ }^{2} / \eta_{\mathrm{q}}{ }^{2}\right)
$$

where $\mathrm{Q}$ represents fluorescence quantum yield, $\mathrm{I}$ is the measured integrated emission intensity, and $\mathrm{A}$ and $\eta$ represent the $332 \mathrm{~nm}$ absorption value and the refractive index of the solvent respectively. " $c$ " and " $q$ " represent the NPs and the standard, respectively.

\subsection{Cell cytotoxicity Assay}

The cell cytotoxicity of STC-CDs was analysed using RAW 264.7 cells in a CCK-8 assay. The suspension of well-cultured cells was diluted to $1 \times 10^{5}$ cells $/ \mathrm{mL}$ and spread on a 96-well plate. PBS buffer was added to the surrounding edges, then the cells were incubated in a $\mathrm{CO}_{2}$ incubator $\left(37^{\circ} \mathrm{C}, 5 \%\right.$ $\mathrm{CO}_{2}$ ) for $24 \mathrm{~h}$. Then, the supernatant was removed from the 96-well plate. Dulbecco's modified Eagle's medium (DMEM) was added to the control group, and samples of different concentrations were added to the drug groups with $100 \mu \mathrm{L}$ per well. The 96-well plate was cleaned three times with PBS then incubated in the $\mathrm{CO}_{2}$ incubator for an additional $24 \mathrm{~h}$ under the same conditions. CCK-8 reagent (10 $\mu \mathrm{L}$ per well) was added to the cultures in the $\mathrm{CO}_{2}$ incubator and incubated for $3 \mathrm{~h}$. Absorbance at $450 \mathrm{~nm}$ was detected with a microplate reader (Biotek, Vermont, USA). The obtained data were calculated according to the following formula:

$$
\text { Cell Viability }(\% \text { of control })=(\mathrm{Ae}-\mathrm{Ab}) /(\mathrm{Ac}-\mathrm{Ab}) \times 100
$$


$\mathrm{Ae}, \mathrm{Ac}$ and $\mathrm{Ab}$ represent the absorbance of the drug administration group, control group and bank group, respectively, at $450 \mathrm{~nm}$.

\subsection{Coagulation Parameter Measurements}

A total of 50 rats were randomly divided into the following five groups (ten per group): negative control group (NS), positive drug group (0.67 KU/kg (HC)), and the high-, medium- and low-dose STC-NP groups (16.67, 8.33, and $4.17 \mathrm{mg} / \mathrm{kg}$, respectively). Rats were anaesthetised with a $4 \%$ pentobarbital sodium $(50 \mathrm{mg} / \mathrm{kg}$ ) by intraperitoneal injection. Abdominal aortic blood was injected into prepacked 3.2\% sodium citrate (blood : citrate : $1: 9, v / v$ ) centrifuge tubes and allowed to react for at least $30 \mathrm{~min}$. Then, the samples were centrifuged at $750 \times \mathrm{g}$ for $15 \mathrm{~min}$ to obtain the supernatant. APTT, TT, PT and FIB were measured using an automatic coagulation analyser [42]. PLT was determined by collecting another $60 \mu \mathrm{L}$ of EDTA-K2 anticoagulant-mixed whole blood.

\subsection{Statistical Analysis}

All experimental data were analysed using the statistical package for the social sciences (SPSS, version 19.0). Normally-distributed and variance homogeneity data were expressed as the mean \pm standard deviation (SD). One-way analysis of variance (ANOVA) was used for pairwise comparisons among multiple experimental groups, and non-normally distributed data were expressed as medians (quartile ranges). $p<0.05$ and $p<0.01$ indicated statistically significant differences.

\section{Conclusions}

This study is the first to report that novel NPs derived from ST have haemostatic effects in mouse tail amputation and liver scratch models, and that these effects may be associated with the exogenous coagulation pathway and activation of the brinogen system, according to the evaluation of the mouse coagulation parameters. The low toxicity of STC-NPs indicated that they have good prospects for biological application. The haemostatic effect of STC-NPs suggests a new approach for the research and development of therapeutic drugs for haemorrhagic diseases, as well as a novel means by which to explore the mechanism of effective TCM ingredients.

Author Contributions: Experiments were designed by Y.Z. (Yusheng Zhao) and H.Q. and conducted by Y.Z. (Yusheng Zhao), J.L., M.Z., J.C. and H.K. Data was analysed by Y.Z. (Yusheng Zhao), Y.Z., and J.C. The manuscript was prepared by Y.Z. and edited by H.Q. All authors read and approved the final manuscript.

Funding: This work is supported by the National Natural Science Foundation (grant number 81573573) and the Classical Prescription Basic Research Team of the Beijing University of Chinese Medicine.

Conflicts of Interest: No conflict of interest was reported by the authors.

\section{References}

1. Chen, C.; Yang, F.Q.; Zhang, Q.; Wang, F.Q.; Hu, Y.J.; Xia, Z.N. Natural Products for Antithrombosis. Evid. Based Complement. Alternat. Med. 2015, 2015, 876426. [CrossRef]

2. Chen, C.; Wang, F.; Xiao, W.; Xia, Z.; Hu, G.; Wan, J.; Yang, F. Effect on platelet aggregation activity: Extracts from 31 Traditional Chinese Medicines with the property of activating blood and resolving stasis. J. Tradit. Chin. Med. 2017, 37, 64-75.

3. Gao, M.; Bao, B.; Cao, Y.; Shan, M.; Cheng, F.; Jiang, M.; Chen, P.; Zhang, L. Chemical Property Changes and Thermal Analysis during the Carbonizing Process of the Pollen Grains of Typha. Molecules 2018, 24, 128. [CrossRef]

4. Qian, Z.Z.; Dan, Y.; Liu, Y.Z.; Peng, Y.J.C.H.M. Pharmacopoeia of the People's Republic of China (2010 Edition): A Milestone in Development of China's Healthcare. China Med. Sci. Technol. Press 2010, 2, 157-160.

5. Zhang, M.; Zhao, Y.; Cheng, J.; Liu, X.; Wang, Y.; Yan, X.; Zhang, Y.; Lu, F.; Wang, Q.; Qu, H. Novel carbon dots derived from Schizonepetae Herba Carbonisata and investigation of their haemostatic efficacy. Artif. Cells Nanomed. Biotechnol. 2018, 46, 1562-1571. [CrossRef] [PubMed] 
6. Zhao, Y.; Guo, J.; Liu, T.; Li, C.; Cao, C.; Yi, Y.; Hao, R.; Li, R. Pharmacology experimental study of new hematischesis compounds after Flos Sophorae carbonized. Zhongguo Zhong Yao Za Zhi 2010, 35, 2346-2349. [PubMed]

7. Chen, Y.; Chen, Q.; Wang, X.; Sun, F.; Fan, Y.; Liu, X.; Li, H.; Deng, Z. Hemostatic action of lotus leaf charcoal is probably due to transformation of flavonol aglycons from flavonol glucosides in traditional Chinses medicine. J. Ethnopharmacol. 2019, 249, 112364. [CrossRef] [PubMed]

8. Zhang, Q.; Wang, Y.L.; Gao, D.; Cai, L.; Yang, Y.Y.; Hu, Y.J.; Yang, F.Q.; Chen, H.; Xia, Z.N. Comparing coagulation activity of Selaginella tamariscina before and after stir-frying process and determining the possible active constituents based on compositional variation. Pharm. Biol. 2018, 56, 67-75. [CrossRef]

9. Mishra, V.; Patil, A.; Thakur, S.; Kesharwani, P. Carbon dots: Emerging theranostic nanoarchitectures. Drug Discov. Today 2018, 23, 1219-1232. [CrossRef]

10. Li, G.-L.; Wei, S.-H.; Zhang, Z.-L.; Li, H.-B. Comparison of Contents of Total Flavonoids in Different Processed Products of Selaginella. China J. Chin. Med. 2011, 26, 194-195.

11. Zhang, F.; Zhang, M.; Zheng, X.; Tao, S.Y.; Zhang, Z.Q.; Sun, M.D.; Song, Y.B.; Zhang, J.; Shao, D.; He, K.; et al. Berberine-based carbon dots for selective and safe cancer theranostics. RSC Adv. 2018, 8, 1168-1173. [CrossRef]

12. Liu, X.; Wang, Y.; Yan, X.; Zhang, M.; Zhang, Y.; Cheng, J.; Lu, F.; Qu, H.; Wang, Q.; Zhao, Y. Novel Phellodendri Cortex (Huang Bo)-derived carbon dots and their hemostatic effect. Nanomedicine 2018, 13, 391-405. [CrossRef] [PubMed]

13. Wei, J.M.; Zhang, X.; Sheng, Y.Z.; Shen, J.M.; Huang, P.; Guo, S.K.; Pan, J.Q.; Liu, B.T.; Feng, B.X. Simple one-step synthesis of water-soluble fluorescent carbon dots from waste paper. New J. Chem. 2014, 38, 906-909. [CrossRef]

14. Zhu, C.Z.; Zhai, J.F.; Dong, S.J. Bifunctional fluorescent carbon nanodots: Green synthesis via soy milk and application as metal-free electrocatalysts for oxygen reduction. Chem. Commun. 2012, 48, 9367-9369. [CrossRef]

15. Jahanbakhshi, M.; Habibi, B. A novel and facile synthesis of carbon quantum dots via salep hydrothermal treatment as the silver nanoparticles support: Application to electroanalytical determination of $\mathrm{H} 2 \mathrm{O} 2$ in fetal bovine serum. Biosens. Bioelectron. 2016, 81, 143-150. [CrossRef]

16. Liu, L.; Chen, L.; Liang, J.; Liu, L.; Han, H. A Novel Ratiometric Probe Based on Nitrogen-Doped Carbon Dots and Rhodamine B Isothiocyanate for Detection of Fe(3+) in Aqueous Solution. J. Anal. Methods Chem. 2016, 2016, 4939582. [CrossRef]

17. Zhang, J.H.; Niu, A.; Li, J.; Fu, J.W.; Xu, Q.; Pei, D.S. In vivo characterization of hair and skin derived carbon quantum dots with high quantum yield as long-term bioprobes in zebrafish. Sci. Rep. 2016, 6, 37860. [CrossRef]

18. Mewada, A.; Pandey, S.; Shinde, S.; Mishra, N.; Oza, G.; Thakur, M.; Sharon, M.; Sharon, M. Green synthesis of biocompatible carbon dots using aqueous extract of Trapa bispinosa peel. Mater. Sci. Eng. C 2013, 33, $2914-2917$. [CrossRef]

19. Saha, A.K.; Sharma, P.; Sohn, H.B.; Ghosh, S.; Das, R.K.; Hebard, A.F.; Zeng, H.; Baligand, C.; Walter, G.A.; Moudgil, B.M. Fe Doped CdTeS Magnetic Quantum Dots for Bioimaging. J. Mater. Chem. B 2013, 1, 6312-6320. [CrossRef]

20. Li, Y.; Li, S.; Wang, Y.; Wang, J.; Liu, H.; Liu, X.; Wang, L.; Liu, X.; Xue, W.; Ma, N. Electrochemical synthesis of phosphorus-doped graphene quantum dots for free radical scavenging. Phys. Chem. Chem. Phys. 2017, 19, 11631-11638. [CrossRef]

21. Shen, J.; Shang, S.M.; Chen, X.Y.; Wang, D.; Cai, Y. Highly fluorescent N, S-co-doped carbon dots and their potential applications as antioxidants and sensitive probes for $\mathrm{Cr}$ (VI) detection. Sens. Actuators B Chem. 2017, 248, 92-100. [CrossRef]

22. Liu, J.; Lu, S.; Tang, Q.; Zhang, K.; Yu, W.; Sun, H.; Yang, B. One-step hydrothermal synthesis of photoluminescent carbon nanodots with selective antibacterial activity against Porphyromonas gingivalis. Nanoscale 2017, 9, 7135-7142. [CrossRef] [PubMed]

23. Li, L.S.; Jiao, X.Y.; Zhang, Y.; Cheng, C.; Huang, K.; Xu, L. Green synthesis of fluorescent carbon dots from Hongcaitai for selective detection of hypochlorite and mercuric ions and cell imaging. Sens. Actuators B Chem. 2018, 263, 426-435. [CrossRef] 
24. Wang, K.; Gao, Z.; Gao, G.; Wo, Y.; Wang, Y.; Shen, G.; Cui, D. Systematic safety evaluation on photoluminescent carbon dots. Nanos. Res. Lett. 2013, 8, 122. [CrossRef]

25. Pereira, G.; Monteiro, C.A.P.; Albuquerque, G.M.; Pereira, M.I.A.; Cabrera, M.P.; Cabral, P.E.; Pereira, G.A.L.; Fontes, A.; Santos, B.S. (Bio)conjugation Strategies Applied to Fluorescent Semiconductor Quantum Dots. J. Braz. Chem. Soc. 2019, 30, 2536-2560. [CrossRef]

26. Feng, T.; Ai, X.; An, G.; Yang, P.; Zhao, Y. Correction to Charge-Convertible Carbon Dots for Imaging-Guided Drug Delivery with Enhanced in Vivo Cancer Therapeutic Efficiency. ACS Nano 2016, 10, 4410-4420. [CrossRef]

27. Sahu, S.; Behera, B.; Maiti, T.K.; Mohapatra, S. Simple one-step synthesis of highly luminescent carbon dots from orange juice: Application as excellent bio-imaging agents. Chem. Commun. 2012, 48, 8835-8837. [CrossRef]

28. Tu, X.L.; Ma, Y.F.; Cao, Y.H.; Huang, J.; Zhang, M.X.; Zhang, Z.J. PEGylated carbon nanoparticles for efficient in vitro photothermal cancer therapy. J. Mater.Chem. B 2014, 2, 2184-2192. [CrossRef]

29. Yan, X.; Zhao, Y.; Luo, J.; Xiong, W.; Liu, X.; Cheng, J.; Wang, Y.; Zhang, M.; Qu, H. Hemostatic bioactivity of novel Pollen Typhae Carbonisata-derived carbon quantum dots. J. Nanobiotechnol. 2017, 15, 60. [CrossRef]

30. Luo, J.; Zhang, M.L.; Cheng, J.J.; Wu, S.H.; Xiong, W.; Kong, H.; Zhao, Y.; Qu, H.H. Hemostatic effect of novel carbon dots derived from Cirsium setosum Carbonisata. RSC Adv. 2018, 8, 37707-37714. [CrossRef]

31. Cheng, J.J.; Zhang, M.L.; Sun, Z.W.; Lu, F.; Xiong, W.; Luo, J.; Kong, H.; Wang, Q.G.; Qu, H.H.; Zhao, Y. Hemostatic and hepatoprotective bioactivity of Junci Medulla Carbonisata-derived Carbon Dots. Nanomedicine 2019, 14, 431-446. [CrossRef] [PubMed]

32. Himaja, A.L.; Karthik, P.S.; Singh, S.P. Carbon Dots: The Newest Member of the Carbon Nanomaterials Family. Chem. Rec. 2015, 15, 595-615. [CrossRef] [PubMed]

33. Xin, T.Z.; Ananthanarayanan, A.; Luo, K.Q.; Peng, C.J.S. Glowing Graphene Quantum Dots and Carbon Dots: Properties, Syntheses, and Biological Applications. Small. 2014, 11, 1620-1636.

34. Yang, Y.; Wang, X.; Liao, G.; Liu, X.; Chen, Q.; Li, H.; Lu, L.; Zhao, P.; Yu, Z. iRGD-decorated red shift emissive carbon nanodots for tumor targeting fluorescence imaging. J. Colloid Interface Sci. 2018, 509, 515-521. [CrossRef]

35. Zhu, J.Y.; Dong, Y.; Zhang, S.; Fan, Z.J. Application of Carbon-/Graphene Quantum Dots for Supercapacitors. Acta Phys. Chim. Sin. 2020, 36, 1-16.

36. Pramanik, A.; Biswas, S.; Kumbhakar, P. Solvatochromism in highly luminescent environmental friendly carbon quantum dots for sensing applications: Conversion of bio-waste into bio-asset. Spectrochim. Acta A Mol. Biomol. Spectrosc. 2018, 191, 498-512. [CrossRef]

37. Zheng, X.K.; Zhang, L.; Wang, W.W.; Wu, Y.Y.; Zhang, Q.B.; Feng, W.S. Anti-diabetic activity and potential mechanism of total flavonoids of Selaginella tamariscina (Beauv.) Spring in rats induced by high fat diet and low dose STZ. J. Ethnopharmacol. 2011, 137, 662-668. [CrossRef]

38. Furie, B.; Furie, B.C. Mechanisms of thrombus formation. N. Engl. J. Med. 2008, 359, 938-949. [CrossRef]

39. Xin, N.; Li, Y.J.; Li, Y.; Dai, R.J.; Meng, W.W.; Chen, Y.; Schlappi, M.; Deng, Y.L. Dragon's Blood extract has antithrombotic properties, affecting platelet aggregation functions and anticoagulation activities. J. Ethnopharmacol. 2011, 135, 510-514. [CrossRef]

40. Fan, H.J.; Mei, Z.; Yang, J.; Cao, Y.; Wang, Q.J.R. Study on HPLC Fingerprint Chromatogram of Selaginella tamariscina and Selaginella pulvinate and Amentoflavone Determination. Res. Pract. Chin. Med. 2011, 3, 31-33.

41. Ding, C.; Cao, X.; Zhang, C.; He, T.; Hua, N.; Xian, Y. Rare earth ions enhanced near infrared fluorescence of Ag2S quantum dots for the detection of fluoride ions in living cells. Nanoscale 2017, 9, 14031-14038. [CrossRef] [PubMed]

42. Vanblerk, M.; Bailleul, E.; Chatelain, B.; Demulder, A.; Devreese, K.; Douxfils, J.; Jacquemin, M.; Jochmans, K.; Mullier, F.; Wijns, W. Influence of apixaban on commonly used coagulation assays: Results from the Belgian national External Quality Assessment Scheme. Int. J. Lab. Hematol. 2017, 39, 402-408. [CrossRef] [PubMed]

(C) 2020 by the authors. Licensee MDPI, Basel, Switzerland. This article is an open access article distributed under the terms and conditions of the Creative Commons Attribution (CC BY) license (http://creativecommons.org/licenses/by/4.0/). 